\title{
CAN WE PREDICT STEERING CONTROL PERFORMANCE FROM A 2D SHAPE DETECTION TASK?
}

\author{
Bobby Nguyen $^{1}$, Yan Zhuo ${ }^{2}$ \& Rui $\mathrm{Ni}^{1}$ \\ ${ }^{1}$ Wichita State University, Wichita, Kansas, USA \\ ${ }^{2}$ Institute of Biophysics, Chinese Academy of Sciences, Beijing, China \\ Email: bhnguyen@wichita.edu
}

\begin{abstract}
Summary: Research has shown the importance of spatial and temporal integration of visual information in motion perception and steering control under reduced visibility conditions. The current study examined the relationship between a 2D shape detection task and a steering control task under reduced visibility conditions for younger drivers. In the $2 \mathrm{D}$ shape detection task, the spatial and temporal characteristics, and the contrast of the stimuli were manipulated by varying the number, the lifetime, and the contrast of the random dots. In the steering task, the visibility of the driving scene was manipulated by varying the quantity and quality of the optical flow information. We found that the correlation between shape detection task and steering control task under low contrast conditions depended on temporal integration. These results suggest that under reduced visibility conditions, temporal integration of visual information may play a larger role than spatial integration.
\end{abstract}

\section{INTRODUCTION}

A critical perceptual task during driving is to use visual information to steer the vehicle. Failure to accurately detect changes in the visual environment and make corrections to the vehicle's path of motion could cause serious consequences for driver safety. One source of visual information that has been extensively studied for the control of steering is optical flow - the perspective transformation of the optic array (Gibson, 1966; 1979). However, when the visual information is impoverished (e.g., in foggy or rainy weather conditions, or when driving at night), the processing of optical flow could be a challenging task for drivers. Previous research has found that the presence of fog may lead to higher injury and death rates in accidents (AlGhamdi, 2007). Indeed, recent driving simulation studies have provided evidence of decreased driving performance under simulated fog conditions. It was found that foggy conditions not only decreased the car following performance for both younger (Kang, Ni, \& Andersen, 2008) and older drivers (Ni, Kang, \& Andersen, 2010), but also adversely affected the detection of imminent collision events (Ni, Bian, Guindon, \& Andersen, 2012). A recent driving simulator study systematically investigated steering control (referred to as SC) performance under low visibility conditions (Nguyen, Zhuo, \& Ni, 2011). They examined the effect of optical flow quality (i.e., contrast) and optical flow quantity (i.e., number of dots in the optical flow field) on SC performance. It was found that decreases in the quality and quantity of optical flow had detrimental effects on SC performance for both younger and older drivers, with the effect more pronounced for older drivers.

The process of optical flow information requires observers to integrate visual information over space and time. Nguyen and colleagues (Nguyen, Zhuo, \& Ni, 2011) showed the importance of 
spatial integration (i.e., optical flow quantity) and the quality of information for SC. This is consistent with a previous finding that age-related declines in the processing of 2D optical flow information result from spatial integration (Andersen \& Ni, 2008). However, it was not clear until recently how spatial and temporal integration was affected by low visibility conditions (Nguyen \& Ni, 2012). Nguyen and Ni examined the effect of contrast on 2D shape perception (referred to as 2DSP) resulted from kinetic occlusion information. Their results showed decreased performance as a function of decreased contrast, decreased temporal integration, and decreased spatial integration.

Since the aforementioned studies showed the effect of optical flow quantity and quality on SC performance, and the role of temporal and spatial integration in the processing of optical flow information, we hypothesize that a 2DSP task that measures spatial and temporal integration of optic flow information could potentially predict SC performance under low visibility conditions. In the current study, we examined three factors that are involved in the processing of optical flow information for both 2DSP and SC. The first factor involves spatial information, which was manipulated by changing the quantity (i.e., the amount) of stimuli dots. The second factor involves temporal information, which was manipulated by changing the lifetime of stimuli dots. The last factor regarding the quality of visual information was manipulated by changing the contrast of dots. Three separate experiments were conducted in which participants' perceptual thresholds in each of the three factors were measured and correlated with SC performance. If our hypothesis is correct, then performance on the 2DSP task will be positively correlated to SC performance. If performance on the 2DSP task is related to SC performance, it may be possible to utilize the 2DSP task as a predictor for SC performance, or even broader, driving performance. Further, given a positive relationship, it may be possible to improve driving performance using the 2DSP task as a training tool.

\section{EXPERIMENTS}

\section{General Methods}

Participants. In each of the three experiments, there were seven participants (mean age $=25.4$, 21.6, 22.1, respectively). All participants had a valid driver's license, had normal or corrected-tonormal vision and were naïve to the purpose of the study.

Procedure. Participants in each of the three conditions performed their respective 2DSP task and a steering control task. The order in which the 2DSP and steering control task was administered was counterbalanced across participants.

\section{Shape Detection Tasks}

Apparatus. The displays were presented on a 20-inch CRT monitor with a visual angle of $39^{\circ}$ by $30^{\circ}$ at a refresh rate of $60 \mathrm{~Hz}$ and a resolution of $800 \times 600$. Drivers viewed the displays binocularly at a distance of $57 \mathrm{~cm}$ from the screen in a darkened room, with their chin on a chin rest. A four-button keyboard was used to input responses.

Stimuli. The stimuli were similar to those used in Andersen \& Ni’s (2008) study. The stimuli 
were computer generated displays of white dots $\left(0.10^{\circ}\right.$ by $\left.0.10^{\circ}\right)$ projected onto an opaque gray object and gray background with a luminance of $7.0 \mathrm{~cd} / \mathrm{m}^{2}$. The opaque object is invisible unless it moves on the background. Four different object shapes were presented - square, circle, diamond, or triangle. The objects were positioned in the center of the display, and randomly moved from the center to the right or left, then back to the center at a velocity of $8.5 \%$ sec. Only dots projected on the object moved while the background dots remained stationary. Both dots on the object and the background disappeared and were randomly repositioned at the end of their lifetime (e.g., 3 frames, or $50 \mathrm{~ms}$ ). Display duration was $5 \mathrm{~s}$ for each trial.

Design. There were three variables of interest: dot density, dot contrast, and dot lifetime. Each experiment utilized a different combination in which one variable was measured as the dependent variable while the other two variables were manipulated as independent variables (see Table 1 for a complete list of combinations).

Table 1. Independent and Dependent Variables of Experiments

\begin{tabular}{cccc}
\hline & \multicolumn{3}{c}{ Variables } \\
\cline { 2 - 4 } Experiment & Dot density & Dot contrast & Dot lifetime \\
\hline 1 & DV & IV & IV \\
\hline 2 & IV & DV & IV \\
\hline 3 & IV & IV & DV \\
\hline *DV: Dependent Variable; IV: Independent Variable
\end{tabular}

In Experiments 1, 2, and 3, the dependent variables were dot density threshold (in dots/degree ${ }^{2}$ ), dot contrast threshold (Michelson contrast), and dot lifetime threshold (in ms), respectively. Dot density independent variables were comprised of two levels, low and high, 0.67 and 1.04 dots/degree ${ }^{2}$, respectively. Dot contrast independent variables were comprised of two levels, low and high, .10 and .26 Michelson contrast, respectively. Dot lifetime independent variables were comprised of two levels, low and high, 33 and 100 ms, respectively. All independent variables were treated as within-subjects variables.

A 3-down, 1-up staircase was used to measure threshold levels for each dependent variable. A staircase is an adaptive psychophysical method used to measure sensory thresholds. It has been found to be more efficient than other psychophysical methods (Cornsweet, 1962). To illustrate the staircase, for example, in Experiment 1, the threshold of dot density was measured in each of the four conditions ( 2 dot contrast x 2 dot lifetime). A participant must obtain three correct trials (i.e., correctly identify three shapes) in a row before the dot density is decreased in the next trial, while one incorrect trial results in an increase of dot density in the subsequent trial. The threshold value indicates the minimum dot density that is required for the participant to correctly identify a shape, under the combined conditions of the other two independent variables. For all three experiments, higher thresholds indicate worse performance on 2DSP.

Procedure. Participants were informed they would be shown a series of displays of one of the four moving objects - a square, diamond, circle, or triangle. The participant's task was to identify which shape they perceived in the display. The participant's accuracy was recorded to adjust the staircase. Participants used a 4-button keyboard to indicate their answer. Following a practice block consisting of 60 trials, participants were tested on their thresholds once for each of 
the four conditions (2 IV1 conditions x 2 IV2 conditions) in a random order. Auditory feedback in the form of a beep indicated incorrect answers.

\section{Steering Control Task}

Apparatus. The displays were presented on a 46-inch LCD monitor with a refresh rate at $60 \mathrm{~Hz}$ and a resolution at 1920 x 1080 . Drivers viewed the displays binocularly at a distance of approximately $215 \mathrm{~cm}$ from the screen and in a darkened room. A Logitech Driving Force GT steering wheel system was used for steering input.

Stimuli. The displays simulated driving through a 3-D array of dots located on the ground plane. The simulated speed was $72 \mathrm{~km} / \mathrm{h}$. The dimensions of the space were $400 \mathrm{~m}$ in width and $150 \mathrm{~m}$ in depth, with the simulated eye position of the driver at $1.6 \mathrm{~m}$ above the ground. The horizontal position of drivers was perturbed according to a sum of three prime sine-wave frequencies function. Amplitudes for each frequency were selected to provide equal energy in the Fourier domain. The three frequencies used were $0.083,0.161$, and $0.216 \mathrm{~Hz}$. The amplitudes for these frequencies were 17.51, 6.74, and $9.02 \mathrm{~m}$, respectively. The first two phases of the sine-wave function were randomized across trials. The last phase was determined such that the output of the sum of the three sine-wave functions was zero at the beginning of each trial. The dots were randomly positioned at the beginning of each trial, and then the viewpoint moved forward. Dots that moved out of the view point were randomly repositioned at the far end of the ground plane. Display duration for each trial was 70 seconds. The background had an average luminance of 42 $\mathrm{cd} / \mathrm{m}^{2}$. The luminance of the dots was either $56.8 \mathrm{~cd} / \mathrm{m}^{2}$ or $70.0 \mathrm{~cd} / \mathrm{m}^{2}$ that resulted in a Michelson contrast of .15 or .25 , respectively.

Design. The independent variables for all three experiments were dot contrast (low and high, .15 and .25 Michelson contrast, respectively) and dot density (low and high, 0.06 and 0.30 dots/degree ${ }^{2}$, respectively). All independent variables were treated as within-subjects variables. The dependent variable was a root mean square (RMS) score for each trial, which represented the deviation of vehicle position from the original straight path.

Procedure. Participants were instructed to drive along a straight path while the vehicle's position was subjected to the perturbing forces. Each trial began with a stationary scene. When the participant was ready to start the experiment, they were instructed to press a button on the steering wheel to start moving forward. During the first 10 seconds, there was no lateral perturbation and steering wheel movements did not affect viewer position. This was done to set up the perception of a straight path for the participants. At the end of the 10 seconds, a beep sounded to notify the participant that they needed to steer the wheel to null out the perturbation. At the end of each trial, the scene stopped. Participants ran through four trials (2 contrast conditions x 2 dot density conditions). The order of the trials was randomized for each participant. During active control, perturbing forces and steering wheel displacement data were sampled 60 times per second. 


\section{RESULTS}

In Experiments 1, 2 and 3, dot density, dot contrast, and dot lifetime thresholds were measured for each of the four conditions in the 2DSP task, respectively, while RMS scores were calculated for each of the four conditions in the SC task. Thus, each participant had eight performance measures. An increase in stimuli threshold indicates a decrease in 2DSP performance, while an increase in RMS represents a greater deviation from the straight driving path indicating poorer driving performance.

Steering performance was assessed by calculating RMS steering error in relation to perturbation forces for each driver on each trial in each condition. It was observed in some trials, due to the accumulation of displacement over a boundless driving space, drivers could not return to the original straight path after a certain amount of time. Therefore, an algorithm was developed to distinguish periods of a trial in which a participant became "lost”. For each trial, an overall RMS score was computed for all data points. Then, for each individual data point, a RMS score was computed for each frame in a 31-frame period (15 preceding, current frame, and 15 proceeding). The data point was deleted if the current RMS score was greater than the overall RMS score (about 35\% data was dropped). A final RMS score was computed for the remaining data points.

For each experiment, Pearson correlation coefficients were computed between scores in the four 2DSP task conditions and scores in the four SC task conditions. The Holm's sequential Bonferroni approach was used to control for Type I error across the correlations. The results of the correlational analyses for Experiment 1, 2 and 3, are presented in Tables 1, 2 and 3, respectively. Further analysis showed that in Experiment 3 the low contrast, low density 2DSP task performance was strongly correlated with the low contrast, low density SC task $(\mathrm{r}(5)=.88$, $\mathrm{p}<.01)$, and the low contrast, high density SC condition, $(\mathrm{r}(5)=.95, \mathrm{p}<.01)$. Performance on the low contrast, high density 2DSP task was strongly correlated with the low contrast, low density SC task (r (5) = .96, p <.01), and with the low contrast, high density SC condition ( $\mathrm{r}(5)$ $=.99, \mathrm{p}<.01)$.

Correlations between SC task and 2DSP task under high contrast conditions were not computed because 2DSP thresholds in that condition were constant. In other words, all participants in that condition reached the minimum lifetime threshold (i.e., $33 \mathrm{~ms}$ ) indicating a ceiling effect.

Table 1. Correlations between 2DSP in Experiment 1(density) and steering control task performance

\begin{tabular}{|c|c|c|c|c|c|}
\hline \multirow{3}{*}{\multicolumn{2}{|c|}{ 2DSP Task Conditions }} & \multicolumn{4}{|c|}{ Steering Control Task Conditions } \\
\hline & & \multicolumn{2}{|c|}{ Low Dot Density } & \multicolumn{2}{|c|}{ High Dot Density } \\
\hline & & Low Contrast & High Contrast & Low Contrast & High Contrast \\
\hline Lifetime & Contrast & & & & \\
\hline Low & Low & .08 & 31 & .36 & .08 \\
\hline Low & High & .07 & .33 & .34 & .14 \\
\hline High & Low & .04 & .04 & .19 & .09 \\
\hline High & High & .02 & .23 & .20 & .03 \\
\hline
\end{tabular}


Table 2. Correlations between 2DSP in Experiment 2 (contrast) and steering control task performance

\begin{tabular}{|c|c|c|c|c|c|}
\hline \multirow{3}{*}{\multicolumn{2}{|c|}{ 2DSP Task Conditions }} & \multicolumn{4}{|c|}{ Steering Control Task Conditions } \\
\hline & & \multicolumn{2}{|c|}{ Low Dot Density } & \multicolumn{2}{|c|}{ High Dot Density } \\
\hline & & Low Contrast & High Contrast & Low Contrast & High Contrast \\
\hline Lifetime & Density & & & & \\
\hline Low & Low & -.13 & -.06 & -.02 & -.14 \\
\hline Low & High & .37 & .50 & .43 & .49 \\
\hline High & Low & -.19 & .12 & .09 & -.07 \\
\hline High & High & -.30 & -.14 & -.19 & -.22 \\
\hline
\end{tabular}

Table 3. Correlations between 2DSP in Experiment 3 (lifetime) and steering control task performance

\begin{tabular}{|c|c|c|c|c|c|}
\hline \multirow{3}{*}{\multicolumn{2}{|c|}{ 2DSP Task Conditions }} & \multicolumn{4}{|c|}{ Steering Control Task Conditions } \\
\hline & & \multicolumn{2}{|c|}{ Low Dot Density } & \multicolumn{2}{|c|}{ High Dot Density } \\
\hline & & \multirow{2}{*}{ Low Contrast } & \multirow[t]{2}{*}{ High Contrast } & \multirow[t]{2}{*}{ Low Contrast } & \multirow[t]{2}{*}{ High Contrast } \\
\hline Contrast & Density & & & & \\
\hline Low & Low & $.88 *$ & -.14 & $.95 *$ & .50 \\
\hline Low & High & $.96 *$ & -.08 & $.99 *$ & .41 \\
\hline High & Low & - & - & - & - \\
\hline High & High & - & - & - & - \\
\hline
\end{tabular}

\section{DISCUSSION}

In this study, we examined the relationship between the performance on 2DSP tasks and the performance on a steering control task. We found that of the three 2DSP tasks, the performance on the task that measured temporal integration was positively correlated to the performance on the SC task under low contrast conditions. In other words, drivers who performed the 2DSP task well (i.e., with smaller temporal thresholds) had lower steering error (i.e., RMS error) on the SC task. However, no such correlation was found between the SC tasks and 2DSP tasks measuring spatial integration (i.e., density) under low contrast conditions. Considered together, these results suggest that under low contrast conditions, drivers may be subject to increased accident risk due to a decreased ability to temporally integrate optical flow information for steering control. The temporal characteristic of optical flow information plays a more important role, as compared to the quality or quantity of optical flow, in determining steering control performance for drivers. Past research (Ball, et al., 1993; Owsley, et al., 1991) has suggested visual acuity is not a good predictor for crash risk. The results in the current study warrants further investigation comparing a driver's performance on the 2DSP and crash risk.

The current study adds to the growing body of literature showing the importance of optical flow in self-locomotion (e.g., Larish \& Flach, 1990), shedding light on which characteristics of optical flow may play a larger role in driving, especially under low visibility conditions. Past research has demonstrated that age-related decreased ability in processing visual information may lead to increased risk for older drivers (e.g., Ni, et al., 2007), especially under low visibility driving conditions (e.g., in fog). An important issue for future research is whether this decrement can be minimized or even reversed through training. Andersen et al. (2010) found that training with subthreshold stimuli improved older observers when performing a texture discrimination task. Richards et al. (2006) found that older observers could improve their performance in divided attention through repeated training with the UFOV task. Similarly, improvements in contrast sensitivity through training were found for older adults in a recent study (Phan and Ni, 2011). An 
interesting topic for future research would be to investigate whether older drivers can improve steering control performance through training using the 2D shape detection task.

\section{ACKNOWLEDGMENTS}

This research was supported by WSU URCA grant and NNSF of China grant 90820307.

\section{REFERENCES}

Al-Ghamdi, A. S. (2007). Experimental evaluation of fog warning system. Accident Analysis and Prevention, 39, 1065-1072.

Andersen, G. J., \& Ni., R. (2008). Aging and visual processing: Declines in spatial not temporal integration. Vision Research, 48, 109-118.

Andersen, G. J., Ni, R., Bower, J.D., Watanabe, T. (2010). Perceptual learning, aging, and improved visual performance in early stages of visual processing, Journal of Vision, 10(13):4, 1-13.

Ball, K., Owsley, C., Sloane, M.E., Roenker, D.L., \& Bruni, J.R. (1993). Visual attention problems as a predictor of vehicle crashes among older drivers. Investigative Ophthalmology \& Visual Science, 34(11), 3110-3123.

Cornsweet, T. N. (1962). The Staircase-Method in psychophysics. The American Journal of Psychology, 75(3), 485-491.

Gibson, J. J. (1966). The senses considered as perceptual systems. Boston: Houghton Mifflin.

Gibson, J. J. (1979). The ecological approach to visual perception. Boston: Houghton Mifflin.

Kang, J. J., Ni, R., \& Andersen, G. J. (2008). The effects of reduced visibility from fog on car following performance. Transportation Research Record: Journal of the Transportation Research Board, 2069, 9-15.

Ni, R., Bian, Z., Guindon, A., \& Andersen, G. (2012). Aging and the detection of imminent collision events under simulated fog conditions. Accident Analysis \& Prevention, 49, 525531.

Ni, R., Kang, J., \& Andersen, G. J. (2007). Age-related driving performance: effect of fog under dual-task conditions. Proceedings of the Fourth International Driving Symposium on Human Factors in Driver Assessment, Training and Vehicle Design, 365-370.

Nguyen, B. \& Ni, R. (2012) Effects of contrast on spatial and temporal integration in 2D shape perception from dynamic occlusion. Journal of Vision, 12(9): 885; doi:10.1167/12.9.885.

Nguyen, B., Zhuo, Y., \& Ni, R. (2011). Aging and Steering Control under Reduced Visibility Conditions. Proceedings of the Sixth International Driving Symposium on Human Factors in Driver Assessment, Training and Vehicle Design, 447-453.

Owsley, C., Ball, K., Sloane, M., Roenker, D.L., \& Bruni, J.R. (1991). Visual perceptual/cognitive correlates of vehicle accidents in older drivers. Psychology and Aging, 6, 403-415.

Phan, M., \& Ni, R. (2011). Training older adults' to improve their contrast sensitivity: A possible or impossible task. Journal of Vision, 11(11): 1027; doi:10.1167/11.11.1027.

Richards, E., Bennett, P. J., Sekuler, A. B. (2006). Age related differences in learning with the useful field of view. Vision Research, 46, 4217-4231. 